\title{
Software defines Hardware - Topology of control systems for retractable roofs
}

\author{
Dr. Martin JENNI ${ }^{\mathrm{a}}$, Thomas HERMEKING ${ }^{\mathrm{b}}$ \\ a PFEIFER Systems, Austria \\ Sonnenstrasse 8, 6822 Satteins \\ ${ }^{\mathrm{b}}$ PFEIFER Seil- und Hebetechnik \\ Dr. Karl Lenz Strasse 66, 87700 Memmingen
}

\begin{abstract}
The scientific understanding of tensile technology in late XX century has arguably revolutionized the engineering design and the construction methods of structures with cables, ropes and tension rods. Adding envelope cladding with modern materials like membranes or ETFE foils large wide span structures are now achievable.
\end{abstract}

The sheer size of wide span structures impacts also the electrical control systems required, as the digital world also is moving forward. While the electrical concepts of light systems are already commonly used for wide span structures, the drive systems for retractable roofs are still considered conservative as safety aspects have a high impact on these special drive systems.

Nevertheless, the development of sophisticated communication languages, enhanced safety protocols in combination with outdoor suited hardware allows new control designs for drive systems of retractable roofs. Furthermore, the use of state of the art components like the latest generation of frequency controller and sensors using bus communication allow the development of safe and economical control systems on wide span structures.

Two examples of different control system topologies are presented here with decentralized control topology of the retractable roofs at the SOFI stadium in Inglewood, US and the centralized control system topology at the Al Janoub Stadium in Al Wakrah, Qatar.

SOFI Stadium uses a complete decentralized control system topology. With "Net" based communication protocols and electrical components with "Net" interface" a communication network can be introduced between the active elements on the wide span structure. The freedom provided by the software of choosing the right hardware allows the optimization of the whole system under different topics like available space for electrical components, wiring, accessibility, maintenance and fast installation.

The Al Janoub stadium uses a centralized control topology because of the local concentration of the drives and the extreme environmental conditions in Qatar. Nearly all active components, like motor drives, switches and frequency controllers are installed in two, 12 feet container in $60 \mathrm{~m}$ height below the roof. Despite the centralized topology, focusing on the whole drive system, rotation encoders with net capability are used directly at each drive. Next to an reduced effort in wiring and cabling during installation the use of digital communication between sensors also reduces significantly the influence of electrical distortion in data transmission.

New possibilities in control systems also allow an efficient revitalization of existing drive systems, if needed.

Keywords: Lightweight structures, retractable roofs, control system, control topology, drive systems, safety, communication 